\title{
Normal Approximation to the Distribution of the Estimated Yield Index $S_{p k}$
}

\author{
W. L. PEARN ${ }^{1}$, G. H. LIN ${ }^{2}$ and K. H. WANG ${ }^{3}$ \\ ${ }^{1}$ Department of Industrial Engineering \& Management, National Chiao Tung University; \\ ${ }^{2}$ Department of Communication Engineering, National Penghu Institute of Technology; \\ ${ }^{3}$ Department of Applied Mathematics, National Chung Hsing University, Taiwan, R.O. China
}

\begin{abstract}
Process yield is the most common criterion used in the manufacturing industry for measuring process performance. A measurement index, called $S_{p k}$, has been proposed to calculate the yield for normal processes. The measurement index $S_{p k}$ establishes the relationship between the manufacturing specifications and the actual process performance, which provides an exact measure on process yield. Unfortunately, the sampling distribution of the estimated $S_{p k}$ is mathematically intractable. Therefore, process performance testing cannot be performed. In this paper; we consider a normal approximation to the distribution of the estimated $S_{p k}$, and investigate its accuracy computationally. We compare the critical values calculated from the approximate distribution with those obtained using the standard simulation technique, for various commonly used quality requirements. Extensive computational results are provided and analyzed. The investigation is useful to the practitioners for making decisions in testing process performance based on the yield.
\end{abstract}

Key words: critical value, process yield

\section{Introduction}

Process yield has longtime been a standard criterion used in the manufacturing industry as a common measure on process performance. Process yield is currently defined as the percentage of processed product unit passing the inspection. That is, the product characteristic must fall within the manufacturing tolerance. For product units rejected (nonconformities), additional costs would be incurred to the factory for scrapping or repairing the product. All passed product units are equally accepted by the producer, which requires the factory no additional cost. For processes with two-sided manufacturing specifications, the process yield can be calculated as $\%$ Yield $=F(\mathrm{USL})-F(\mathrm{LSL})$, where USL and LSL are the upper and the lower specification limits, respectively, and $F(\cdot)$ is the cumulative distribution function of the process characteristic. If the process characteristic follows the normal distribution, then the process yield can be alternatively expressed as Yield $\%=\Phi($ USL $\mu) / \sigma]-\Phi[(\mathrm{LSL}-\mu) / \sigma]$, where $\mu$ is the process mean, $\sigma$ is the process standard deviation, and $\Phi(\cdot)$ is the cumulative distribution function of the standard normal distribution $N(0,1)$. 
Table I. Some $S_{p k}$ values and the corresponding nonconformities.

\begin{tabular}{llr}
\hline$S_{p k}$ & Yield & \multicolumn{1}{l}{ PPM } \\
\hline 1.00 & 0.9973002039 & 2699.796 \\
1.10 & 0.9990331517 & 966.848 \\
1.20 & 0.9996817828 & 318.217 \\
1.30 & 0.9999038073 & 96.193 \\
1.33 & 0.9999339267 & 66.073 \\
1.40 & 0.9999733085 & 26.691 \\
1.50 & 0.9999932047 & 6.795 \\
1.60 & 0.9999984133 & 1.587 \\
1.67 & 0.9999994557 & 0.544 \\
1.70 & 0.9999996603 & 0.340 \\
1.80 & 0.9999999334 & 0.067 \\
1.90 & 0.9999999880 & 0.012 \\
2.00 & 0.9999999980 & 0.002 \\
\hline
\end{tabular}

Based on the expression of process yield, Boyles (1994) considered the yield measurement index $S_{p k}$ for normal processes (defined in the following). The index $S_{p k}$ establishes the relationship between the manufacturing specifications and the actual process performance, which provides an exact measure on the process yield. If $S_{p k}=c$, then the process yield can be expressed as \% Yield $=2 \Phi(3 c)-1$. Obviously, there is a one-to-one correspondence between $S_{p k}$ and the process yield. Thus, $S_{p k}$ provides an exact (rather than approximate) measure of the process yield. Table I summarizes the process yield, nonconformities (in PPM) as a function of the measurement index $S_{p k}$, for $S_{p k}=1.00(0.1) 2.00$, including the most commonly-used performance requirements, 1.00, 1.33, 1.50, 1.67, and 2.00. For example, if a process has yield measure $S_{p k}=1.33$, then the corresponding nonconformities is roughly 66 PPM (parts per million).

$$
S_{p k}=\frac{1}{3} \Phi^{-1}\left\{\frac{1}{2} \Phi\left(\frac{\mathrm{USL}-\mu}{\sigma}\right)+\frac{1}{2} \Phi\left(\frac{\mu-\mathrm{LSL}}{\sigma}\right)\right\}
$$

\section{Approximate Distribution of the Estimated $S_{p k}$}

To estimate the yield measurement index $S_{p k}$, we consider the following natural estimator $\hat{S}_{p k}$, where $\bar{X}=\left(\sum_{i=1}^{n} X_{i}\right) / n$, and $S=\left[(n-1)^{-1} \sum_{i=1}^{n}\left(X_{i}-\bar{X}\right)^{2}\right]^{1 / 2}$ are 
the sample mean, and the sample standard deviation, the conventional estimators of $\mu$ and $\sigma$, respectively, which may be obtained from a stable process.

$$
\hat{S}_{p k}=\frac{1}{3} \Phi^{-1}\left\{\frac{1}{2} \Phi\left(\frac{\mathrm{USL}-\bar{X}}{S}\right)+\frac{1}{2} \Phi\left(\frac{\bar{X}-\mathrm{LSL}}{S}\right)\right\} .
$$

The exact distribution of $S_{p k}$ is mathematically intractable. Therefore, testing of the process performance cannot be performed. On the other hand, Lee et al. (2002) obtained a normal approximation to the distribution of $S_{p k}$ using Taylor expansion technique. By taking the first order of the Taylor expansion, it is shown that the estimator can be expressed approximately as:

$$
\hat{S}_{p k} \cong S_{p k}+\frac{1}{6 \sqrt{n}} \frac{W}{\phi\left(3 S_{p k}\right)},
$$

where

$$
\begin{aligned}
W= & \frac{\sqrt{n}\left(S^{2}-\sigma^{2}\right)}{\sigma^{2}}\left\{\frac{\mathrm{USL}-\mu}{2 \sigma} \phi\left(\frac{\mathrm{USL}-\mu}{\sigma}\right)+\frac{\mu-\mathrm{LSL}}{2 \sigma} \phi\left(\frac{\mu-\mathrm{LSL}}{\sigma}\right)\right\} \\
& -\frac{\sqrt{n}(\bar{X}-\mu)}{\sigma}\left\{\phi\left(\frac{\mathrm{USL}-\mu}{\sigma}\right)-\phi\left(\frac{\mu-\mathrm{LSL}}{\sigma}\right)\right\} \\
= & \frac{\sqrt{n}\left(S^{2}-\sigma^{2}\right)}{\sigma^{2}}\left\{\frac{d-(\mu-m)}{2 \sigma} \phi\left(\frac{d-(\mu-m)}{\sigma}\right)\right. \\
& \left.+\frac{d+(\mu-m)}{2 \sigma} \phi\left(\frac{d+(\mu-m)}{\sigma}\right)\right\} \\
& -\frac{\sqrt{n}(\bar{X}-\mu)}{\sigma}\left\{\phi\left(\frac{d-(\mu-m)}{\sigma}\right)-\phi\left(\frac{d+(\mu-m)}{\sigma}\right)\right\} .
\end{aligned}
$$

We note that $W=(\sqrt{n / 2})\left[a\left(S^{2}-\sigma^{2}\right) / \sigma^{2}\right]-\sqrt{n}[b(\bar{X}-\mu) / \sigma]$ for $\mu<m$, and $W=(\sqrt{n / 2})\left[a\left(S^{2}-\sigma^{2}\right) / \sigma^{2}\right]+\sqrt{n}[b(\bar{X}-\mu) / \sigma]$ for $\mu>m$, where $a$ and $b$ are functions of $\mu$ and $\sigma$, as defined in the following, and $\phi$ is the probability density function of the standard normal distribution $N(0,1)$. Thus, the statistic $W$ is normally distributed as $N\left(0, a^{2}+b^{2}\right)$, and the natural estimator $\hat{S}_{p k}$ is approximately distributed as $N\left(\mu_{S}, \sigma_{S}^{2}\right)$, where $E\left(\hat{S}_{p k}\right)=\mu_{S}=S_{p k}$ and $\operatorname{Var}\left(\hat{S}_{p k}\right)=\sigma_{S}^{2}=\left(a^{2}+b^{2}\right)\left\{36 n\left[\phi\left(3 S_{p k}\right)\right]^{2}\right\}^{-1}$, which can be expressed as functions of the widely used precision index $C_{p}=(\mathrm{USL}-\mathrm{LSL}) / 6 \sigma$, and the accuracy index $C_{a}=1-|\mu-m| / d$.

The parameter $C_{p}$ is defined as $C_{p}=(\mathrm{USL}-\mathrm{LSL}) / 6 \sigma$, a function of the process standard deviation, which measures the overall process variation relative to the specification tolerance therefore only reflecting process potential. The parameter $C_{a}$ is defined as $C_{a}=1-|\mu-m| / d$ (see Pearn et al. (1998)), a function of the process mean, which measures the degree of process centering, 
where $m=(\mathrm{USL}+\mathrm{LSL}) / 2$ is the mid- point between the upper and the lower specification limits, and $d=(\mathrm{USL}-\mathrm{LSL}) / 2$ is half of the length of the specification interval. The parameter $C_{a}$ alerts the user if the process mean deviates from its target value. In fact, a mathematical relationship among the three measurements can be established as $\Phi\left(3 S_{p k}\right)=\left\{\Phi\left(3 C_{p} C_{a}\right)+\Phi\left[3 C_{p}\left(2-C_{a}\right)\right]\right\} / 2$.

$$
\begin{aligned}
a & =\frac{1}{\sqrt{2}}\left\{\frac{\mathrm{USL}-\mu}{\sigma} \phi\left(\frac{\mathrm{USL}-\mu}{\sigma}\right)+\frac{\mu-\mathrm{LSL}}{\sigma} \phi\left(\frac{\mu-\mathrm{LSL}}{\sigma}\right)\right\} \\
& =\frac{1}{\sqrt{2}}\left\{\frac{d-(\mu-m)}{\sigma} \phi\left(\frac{d-(\mu-m)}{\sigma}\right)+\frac{d+(\mu-m)}{\sigma} \phi\left(\frac{d+(\mu-m)}{\sigma}\right)\right\} \\
& =\frac{1}{\sqrt{2}}\left\{3 C_{p}\left(2-C_{a}\right) \phi\left(3 C_{p}\left(2-C_{a}\right)\right)+3 C_{p} C_{a} \phi\left(3 C_{p} C_{a}\right)\right\} . \\
b & =\phi\left(\frac{\mathrm{USL}-\mu}{\sigma}\right)-\phi\left(\frac{\mu-\mathrm{LSL}}{\sigma}\right) \\
& =\phi\left(\frac{d-(\mu-m)}{\sigma}\right)-\phi\left(\frac{d+(\mu-m)}{\sigma}\right) \\
& =\phi\left\{3 C_{p}\left(2-C_{a}\right)\right\}-\phi\left(3 C_{p} C_{a}\right) .
\end{aligned}
$$

The probability density function of the approximate distribution, $N\left(\mu_{S}, \sigma_{S}^{2}\right)$, can be expressed as:

$$
\begin{aligned}
f(x)= & \sqrt{\frac{18 n}{\pi}} \frac{\phi\left(3 S_{p k}\right)}{\sqrt{a^{2}+b^{2}}} \exp \left[-\frac{18 n\left(\phi\left(3 S_{p k}\right)\right)^{2}}{a^{2}+b^{2}}\right. \\
& \left.\times\left(x-S_{p k}\right)^{2}\right], \quad-\infty<x<\infty .
\end{aligned}
$$

\section{Calculation of the Critical Values}

The formula of the normal approximation obtained for the distribution of $\hat{S}_{p k}$ is rather complicate, and the calculation is cumbersome to deal with. For the approximation to be useful to the practitioners, we calculate the critical values $c_{o}$ computationally using the Maple-V programming software (see Appendix). Since the critical values $c_{o}$ is a function of the parameters $C_{p}$ and $C_{a}$, we have considered the factor of the two parameters in the calculations to ensure that the critical values obtained are reliable.

\subsection{NORMAL APPROXIMATION}

Tables II(a)-II(e) display the parameters of the process characteristics used in the critical value calculations, covering the most commonly used performance requirements $S_{p k}=1.00$ (capable), 1.33 (satisfactory), 1.50 (good), 1.67 (excellent), and 2.00 (super). Table II(a) summarizes the process characteristics with $C_{p}=$ 
Table $2 a . S_{p k}=1.00$ with $C_{p}=1.0(0.1) 2.0$, the corresponding $\mu, \sigma, C_{a}$, and the calculated $a, b$, and $n \operatorname{Var}\left(\hat{S}_{p k}\right)$. Note: $\phi\left(3 S_{p k}\right)=0.004431848$.

\begin{tabular}{lllllll}
\hline$C_{p}$ & $C_{a}$ & $\mu$ & $\sigma$ & $a$ & \multicolumn{1}{l}{$b$} & $n \operatorname{Var}\left(\hat{S}_{p k}\right)$ \\
\hline 1.0 & 1.000000000 & 15.00000000 & 1.666666667 & 0.018802740 & 0.000000000 & 0.500000072 \\
1.1 & 0.845650984 & 15.77174508 & 1.515151515 & 0.016792051 & -0.007843357 & 0.485784252 \\
1.2 & 0.772993431 & 16.13503285 & 1.388888889 & 0.016414728 & -0.008282188 & 0.478071906 \\
1.3 & 0.713386252 & 16.43306874 & 1.282051282 & 0.016369929 & -0.008317128 & 0.476815000 \\
1.4 & 0.662422888 & 16.68788556 & 1.190476190 & 0.016266525 & -0.008319133 & 0.476704578 \\
1.5 & 0.618261111 & 16.90869445 & 1.111111111 & 0.016366355 & -0.008319213 & 0.476698592 \\
1.6 & 0.579619785 & 17.10190108 & 1.041666667 & 0.016366350 & -0.008319216 & 0.476698431 \\
1.7 & 0.545524504 & 17.27237748 & 0.980392157 & 0.016366349 & -0.008319216 & 0.476698383 \\
1.8 & 0.515217587 & 17.42391207 & 0.925925926 & 0.016366349 & -0.008319216 & 0.476698383 \\
1.9 & 0.488100872 & 17.55949564 & 0.877192982 & 0.016366349 & -0.008319216 & 0.476698383 \\
2.0 & 0.463695828 & 17.68152086 & 0.833333333 & 0.016366349 & -0.008319216 & 0.476698383 \\
\hline & & & & & &
\end{tabular}

Table $2 b . S_{p k}=1.33$ with $C_{p}=1.33,1.4(0.1) 2.3$, the corresponding $\mu, \sigma, C_{a}$, and the calculated $a, b$, and $n \operatorname{Var}\left(\hat{S}_{p k}\right)$. Note: $\phi\left(3 S_{p k}\right)=0.000139285$.

\begin{tabular}{lllllll}
\hline$C_{p}$ & $C_{a}$ & $\mu$ & $\sigma$ & $a$ & \multicolumn{1}{l}{$b$} & $n \operatorname{Var}\left(\hat{S}_{p k}\right)$ \\
\hline 1.33 & 1.000000000 & 15.00000000 & 1.250000000 & 0.000785945 & 0.000000000 & 0.884449647 \\
1.4 & 0.912324580 & 15.43837710 & 1.190476190 & 0.000738619 & -0.000246892 & 0.868419183 \\
1.5 & 0.849520868 & 15.75239566 & 1.111111111 & 0.000725580 & -0.000266999 & 0.855878025 \\
1.6 & 0.796341133 & 16.01829434 & 1.041666667 & 0.000724513 & -0.000267996 & 0.854426361 \\
1.7 & 0.749494830 & 16.25252585 & 0.980392157 & 0.000724459 & -0.000268034 & 0.854343495 \\
1.8 & 0.707856167 & 16.46071917 & 0.925925926 & 0.000724458 & -0.000268034 & 0.854341420 \\
1.9 & 0.670600579 & 16.64699711 & 0.877192982 & 0.000724457 & -0.000268034 & 0.854339345 \\
2.0 & 0.637070550 & 16.81464725 & 0.833333333 & 0.000724457 & -0.000268034 & 0.854339345 \\
2.1 & 0.606733857 & 16.96633072 & 0.793650794 & 0.000724457 & -0.000268034 & 0.854339345 \\
2.2 & 0.579155045 & 17.10422478 & 0.757575758 & 0.000724457 & -0.000268034 & 0.854339345 \\
2.3 & 0.553974391 & 17.23012805 & 0.724637681 & 0.000724457 & -0.000268034 & 0.854339345 \\
\hline
\end{tabular}

1.0(01)2.0, the corresponding $C_{a}$, and $(\mu, \sigma)$ for $S_{p k}=1.00$. Table II(b) summarizes the process characteristics with $C_{p}=1.33,1.4(0.1) 2.3$, the corresponding $C_{a}$, and $(\mu, \sigma)$ for $S_{p k}=1.33$. Table II(c) summarizes the process characteristics with $C_{p}=1.5(0.1) 2.5$, the corresponding $C_{a}$, and $(\mu, \sigma)$ for $S_{p k}=1.50$. Table II(d) summarizes the process characteristics $C_{p}=1.67,1.7(0.1) 2.6$, the corresponding $C_{a}$, and $(\mu, \sigma)$ for $S_{p k}=1.67$. Table II(e) summarizes the process characteristics $C_{p}=2.0(0.1) 3.0$, the corresponding $C_{a}$, and $(\mu, \sigma)$ for $S_{p k}=2.00$. 
Table $2 c . S_{p k}=1.50$ with $C_{p}=1.5(0.1) 2.5$, the corresponding $\mu, \sigma, C_{a}$, and the calculated $a, b$, and $n \operatorname{Var}\left(\hat{S}_{p k}\right)$. Note: $\phi\left(3 S_{p k}\right)=0.000015984$.

\begin{tabular}{lllllll}
\hline$C_{p}$ & $C_{a}$ & $\mu$ & $\sigma$ & $a$ & \multicolumn{1}{l}{$b$} & $n \operatorname{Var}\left(\hat{S}_{p k}\right)$ \\
\hline 1.5 & 1.000000000 & 15.00000000 & 1.111111111 & 0.000101720 & 0.000000000 & 1.124965647 \\
1.6 & 0.906849563 & 15.46575219 & 1.041666667 & 0.000095910 & -0.000030236 & 1.099522577 \\
1.7 & 0.853029665 & 15.73485168 & 0.980392157 & 0.000095365 & -0.000030966 & 1.093046152 \\
1.8 & 0.805624734 & 15.97187633 & 0.925925926 & 0.000095336 & -0.000030991 & 1.092613277 \\
1.9 & 0.763223120 & 16.18388440 & 0.877192982 & 0.000095335 & -0.000030991 & 1.092592546 \\
2.0 & 0.725061959 & 16.37469021 & 0.833333333 & 0.000095335 & -0.000030991 & 1.092592546 \\
2.1 & 0.690535199 & 16.54732401 & 0.793650794 & 0.000095335 & -0.000030991 & 1.092592546 \\
2.2 & 0.659147235 & 16.70426383 & 0.757575758 & 0.000095335 & -0.000030991 & 1.092592546 \\
2.3 & 0.630488660 & 16.84755670 & 0.724637681 & 0.000095335 & -0.000030991 & 1.092592546 \\
2.4 & 0.604218299 & 16.97890851 & 0.694444444 & 0.000095335 & -0.000030991 & 1.092592546 \\
2.5 & 0.580049567 & 17.09975217 & 0.666666667 & 0.000095335 & -0.000030991 & 1.092592546 \\
\hline
\end{tabular}

Table $2 d$. $S_{p k}=1.67$ with $C_{p}=1.7,1.7(0.1) 2.6$, the corresponding $\mu, \sigma, C_{a}$, and the calculated $a$, $b$, and $n \operatorname{Var}\left(\hat{S}_{p k}\right)$. Note: $\phi\left(3 S_{p k}\right)=0.000001414$.

\begin{tabular}{lllllll}
\hline$C_{p}$ & $C_{a}$ & $\mu$ & $\sigma$ & $a$ & \multicolumn{1}{l}{$b$} & $n \operatorname{Var}\left(\hat{S}_{p k}\right)$ \\
\hline 1.67 & 1.000000000 & 15.00000000 & 1.000000000 & 0.000010019 & 0.000000000 & 1.394592848 \\
1.7 & 0.960124663 & 15.19937669 & 0.980392157 & 0.000009753 & -0.000002168 & 1.386824835 \\
1.8 & 0.902865766 & 15.48567117 & 0.925925926 & 0.000009519 & -0.000002740 & 1.363175026 \\
1.9 & 0.855248895 & 15.72375553 & 0.877192982 & 0.000009505 & -0.000002757 & 1.360773093 \\
2.0 & 0.812484428 & 15.93757786 & 0.833333333 & 0.000009504 & -0.000002757 & 1.360508999 \\
2.1 & 0.773794664 & 16.13102668 & 0.793650794 & 0.000009504 & -0.000002757 & 1.360508999 \\
2.2 & 0.738622179 & 16.30688911 & 0.757575758 & 0.000009504 & -0.000002757 & 1.360508999 \\
2.3 & 0.706508171 & 16.46745915 & 0.724637681 & 0.000009504 & -0.000002757 & 1.360508999 \\
2.4 & 0.677070331 & 16.61464835 & 0.694444444 & 0.000009504 & -0.000002757 & 1.360508999 \\
2.5 & 0.649987517 & 16.75006242 & 0.6666666667 & 0.000009504 & -0.000002757 & 1.360508999 \\
2.6 & 0.624987997 & 16.87506002 & 0.641025641 & 0.000009504 & -0.000002757 & 1.360508999 \\
\hline
\end{tabular}

Table III displays the critical values $c_{o}$ computed from the normal approximation. We note that for given fixed performance requirement $S_{p k}$, the differences among those calculated critical values corresponding to various parameter values of $C_{p}$ and $C_{a}$, are sufficiently small and can be neglected. To justify this result, we also calculated the main factor in the variance of the normal approximation, $\left(a^{2}+b^{2}\right)\left\{36\left[\phi\left(3 S_{p k}\right)\right]^{2}\right\}^{-1}$ which determines the magnitude of the critical value $c_{o}$, with different parameter values of $C_{p}$ and $C_{a}$. The results are tabulated in 
Table $2 e . S_{p k}=2.00$ with $C_{p}=2.0(0.1) 3.0$, the corresponding $\mu, \sigma, C_{a}$, and the calculated $a, b$, and $n \operatorname{Var}\left(\hat{S}_{p k}\right)$. Note: $\phi\left(3 S_{p k}\right)=0.000000006$.

\begin{tabular}{lllllll}
\hline$C_{p}$ & $C_{a}$ & $\mu$ & $\sigma$ & $a$ & \multicolumn{1}{l}{$b$} & $n \operatorname{Var}\left(\hat{S}_{p k}\right)$ \\
\hline 2.0 & 1.000000000 & 15.00000000 & 0.833333333 & 0.000000052 & 0.000000000 & 2.086419753 \\
2.1 & 0.934480725 & 15.32759638 & 0.793650794 & 0.000000050 & -0.000000012 & 2.040123457 \\
2.2 & 0.891884461 & 15.5405777 & 0.757575758 & 0.000000050 & -0.000000012 & 2.040123457 \\
2.3 & 0.853105312 & 15.73447344 & 0.724637681 & 0.000000050 & -0.000000012 & 2.040123457 \\
2.4 & 0.817559242 & 15.91220379 & 0.694444444 & 0.000000050 & -0.000000012 & 2.040123457 \\
2.5 & 0.784856872 & 16.07571564 & 0.666666667 & 0.000000050 & -0.000000012 & 2.040123457 \\
2.6 & 0.754670069 & 16.22664966 & 0.641025641 & 0.000000050 & -0.000000012 & 2.040123457 \\
2.7 & 0.726719326 & 16.36640337 & 0.617283951 & 0.000000050 & -0.000000012 & 2.040123457 \\
2.8 & 0.700765064 & 16.49617468 & 0.595238095 & 0.000000050 & -0.000000012 & 2.040123457 \\
2.9 & 0.676600752 & 16.61699624 & 0.574712644 & 0.000000050 & -0.000000012 & 2.040123457 \\
3.0 & 0.654047393 & 16.72976304 & 0.555555556 & 0.000000050 & -0.000000012 & 2.040123457 \\
\hline
\end{tabular}

Tables II(a)-II(e), which indicate that the factor $\left(a^{2}+b^{2}\right)\left\{36\left[\phi\left(3 S_{p k}\right)\right]\right\}^{-1}$ is insensitive to the value changes of $C_{p}$ and $C_{a}$ in all cases, except for $S_{p k}=C_{p}$ ( $C_{a}=1.00$, the process is perfectly centered). Consequently, the critical values $c_{o}$ may be considered as a constant, which is independent of the process characteristics $C_{p}$ and $C_{a}$ for fixed performance requirement $S_{p k}$. Such behavior of the normal approximation can be expected, as it is a linear approximation obtained from taking the first term in the Taylor expansion.

\subsection{SimULATION TECHNIQUE}

To assess the accuracy of the normal approximation, we also obtain the critical values using the standard simulation technique to compare with the critical values obtained from the normal approximation, under the same performance requirements. We note that the natural estimator $S_{p k}$ can be rewritten and expressed as a function of the two parameters $C_{p}$ and $C_{a}$. In fact, a mathematical relationship among the three measurements can be established as $\Phi\left(3 S_{p k}\right)=\left\{\Phi\left(3 C_{p} C_{a}\right)+\right.$ $\left.\Phi\left[3 C_{p}\left(2-C_{a}\right)\right]\right\} / 2$.

$$
\begin{aligned}
S_{p k} & =\frac{1}{3} \Phi^{-1}\left\{\frac{1}{2} \Phi\left(\frac{\mathrm{USL}-\mu}{\sigma}\right)+\frac{1}{2} \Phi\left(\frac{\mu-\mathrm{LSL}}{\sigma}\right)\right\} \\
& =\frac{1}{3} \Phi^{-1}\left\{\frac{1}{2} \Phi\left(\frac{d-|\mu-m|}{\sigma}\right)+\frac{1}{2} \Phi\left(\frac{d+|\mu-m|}{\sigma}\right)\right\} \\
& =\frac{1}{3} \Phi^{-1}\left\{\frac{1}{2} \Phi\left(\frac{1-|\mu-m| / d}{\sigma / d}\right)+\frac{1}{2} \Phi\left(\frac{1+|\mu-m| / d}{\sigma / d}\right)\right\}
\end{aligned}
$$




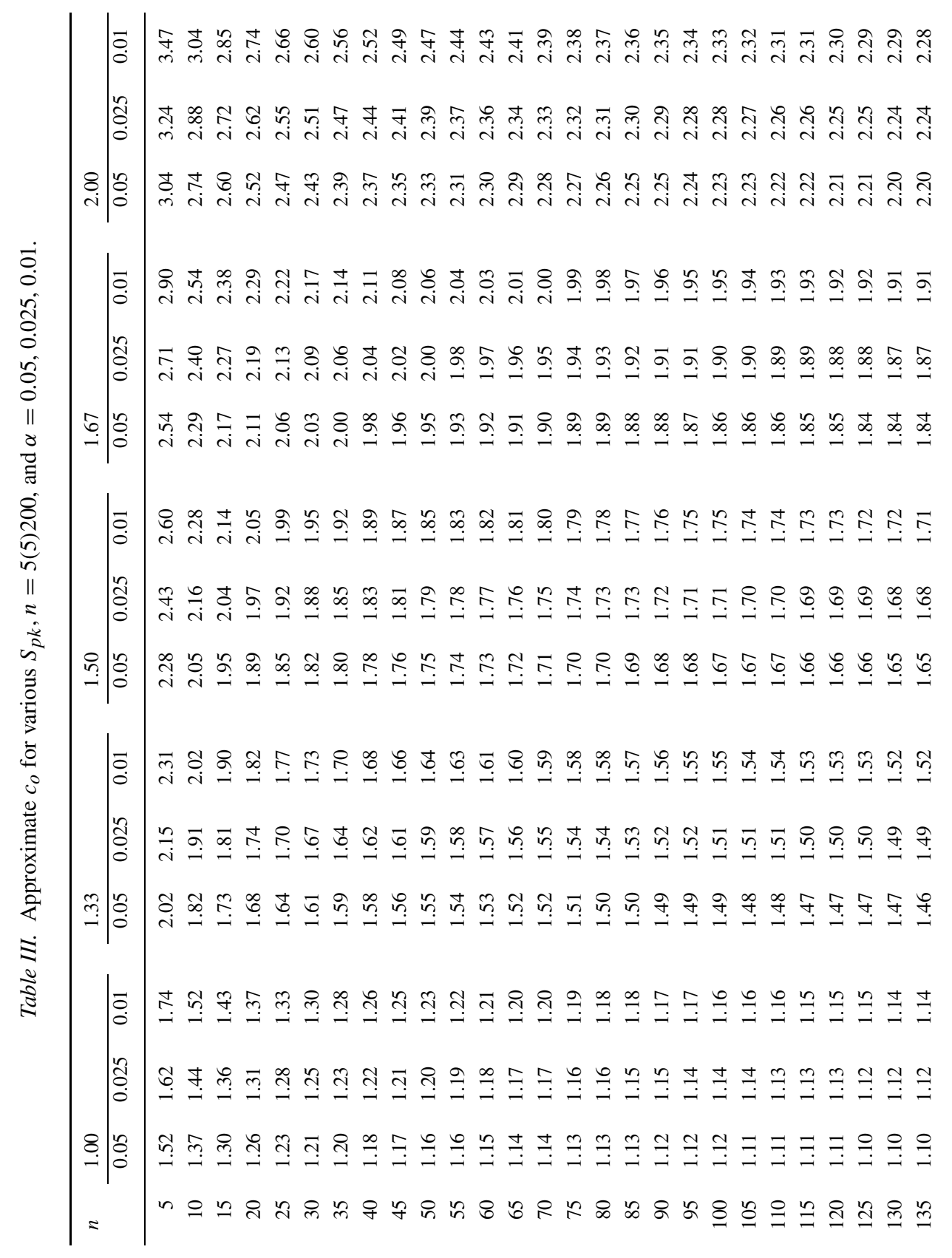




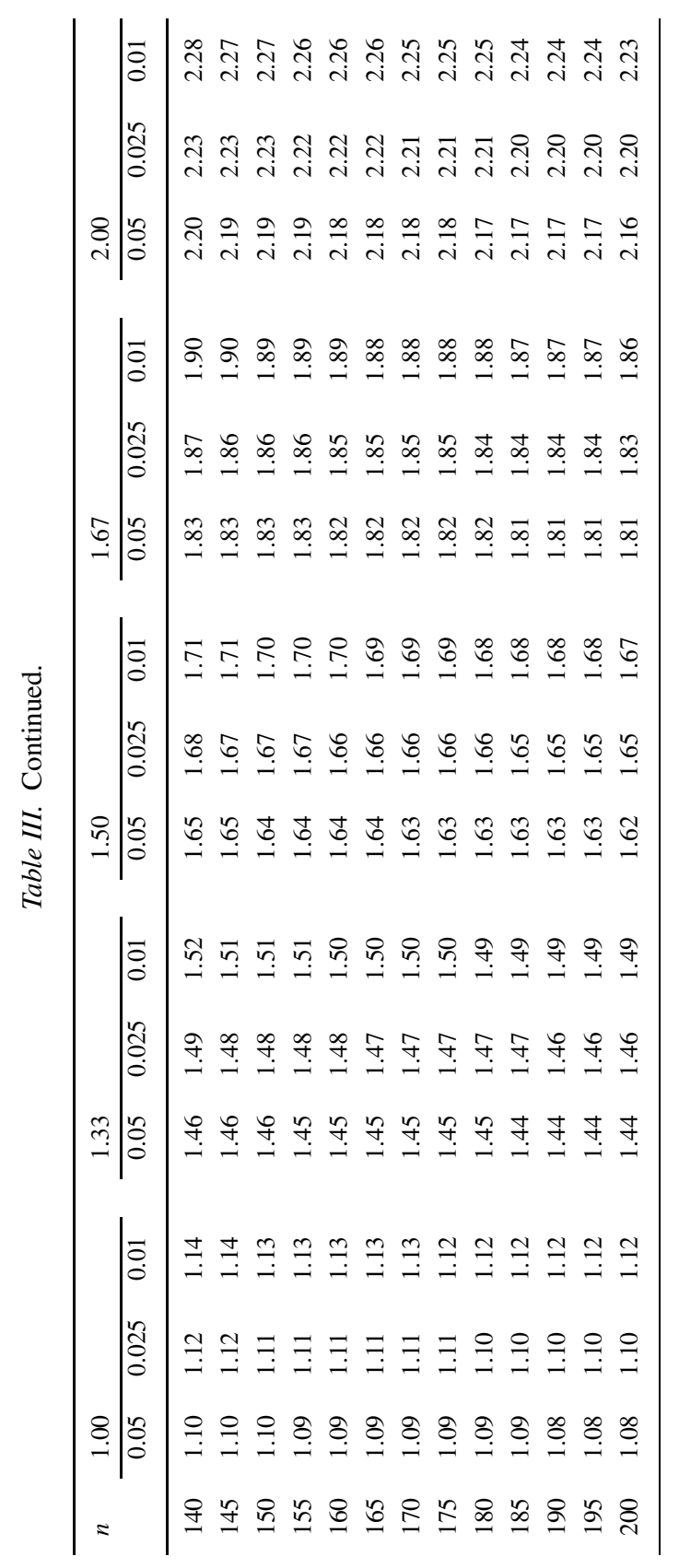




$$
=\frac{1}{3} \Phi^{-1}\left\{\frac{1}{2} \Phi\left[3 C_{p} C_{a}\right]+\frac{1}{2} \Phi\left[3 C_{p}\left(2-C_{a}\right)\right]\right\} .
$$

The simulation was carried out using the SAS programming software, with $N=10000$ replications for each sample size of $n$, on the same set of performance requirements $S_{p k}=1.00,1.33,1.50,1.67$, and 2.00, displayed in Tables II(a)-II(e). The simulation results indicate that the critical values are more sensitive to the two parameters $C_{p}$ and $C_{a}$ than those from the normal approximation. For example, given fixed $S_{p k}=1.67$ with $n=20, c_{o}=2.24$ for $C_{p}=1.67$, and $c_{o}=2.30$ for $C_{p}=1.9$. For practical purpose, we may take the maximal values of $c_{o}$ among those parameters of $C_{p}$ and $C_{a}$ we investigated, to obtain conservative bounds on the critical values for reliability purpose. This approach ensures that the decisions made based on the critical values have the risk of wrongly concluding an incapable process as capable, no greater than the preset type I error $\alpha$. Table IV summarizes the critical values $c_{o}$ (the maximal ones among those with different $C_{p}$ and $C_{a}$ ) obtained from the simulation.

\section{Accuracy of the Normal Approximation}

Table $\mathrm{V}$ displays a comparison of the critical values $c_{o}$ generated by the normal approximation and the simulation technique for various selected sample sizes $n=5(5) 50,60,65,75,90,110,130$, and 150 , with risk $\alpha=0.05$. It is noted that the normal approximation significantly under- approximates the critical values, particularly, for small sample sizes $n<40$, as the magnitude of the underestimation exceeds 0.10 . Therefore, for short run applications (such as accepting a supplier providing short production runs in QS-9000 certification), one should avoid using the normal approximation. It is also noted that the underestimation can be as large as 0.07 for $n=50,0.03$ for $n=110$, and 0.02 for $n=150$. Therefore, in real applications a sample of size greater than 150 is recommended.

\subsection{CONVERGENCE OF THE APPROXIMATION}

Table VI displays the sample sizes required for the normal approximation to converge to $S_{p k}$ within a sampling error less than $0.10,0.09,0.08,0.07,0.06,0.05$, $0.04,0.03,0.02,0.01,0.00$ respectively (with accuracy up to the second decimal point, or $5 \times 10^{-3}$ ). For example, for $S_{p k}=1.33$ with risk $\alpha=0.05$, a sample size of $n \geq 95909$ ensures that the sampling error is no greater than $5 \times 10^{-3}$ which is negligible. Thus, if $\hat{S}_{p k}>1.33$, then we may conclude that the actual performance $S_{p k}>1.33$. The investigation is not for practical purpose. But, the computations illustrate the behavior, and the rate of convergence for the normal approximation. 


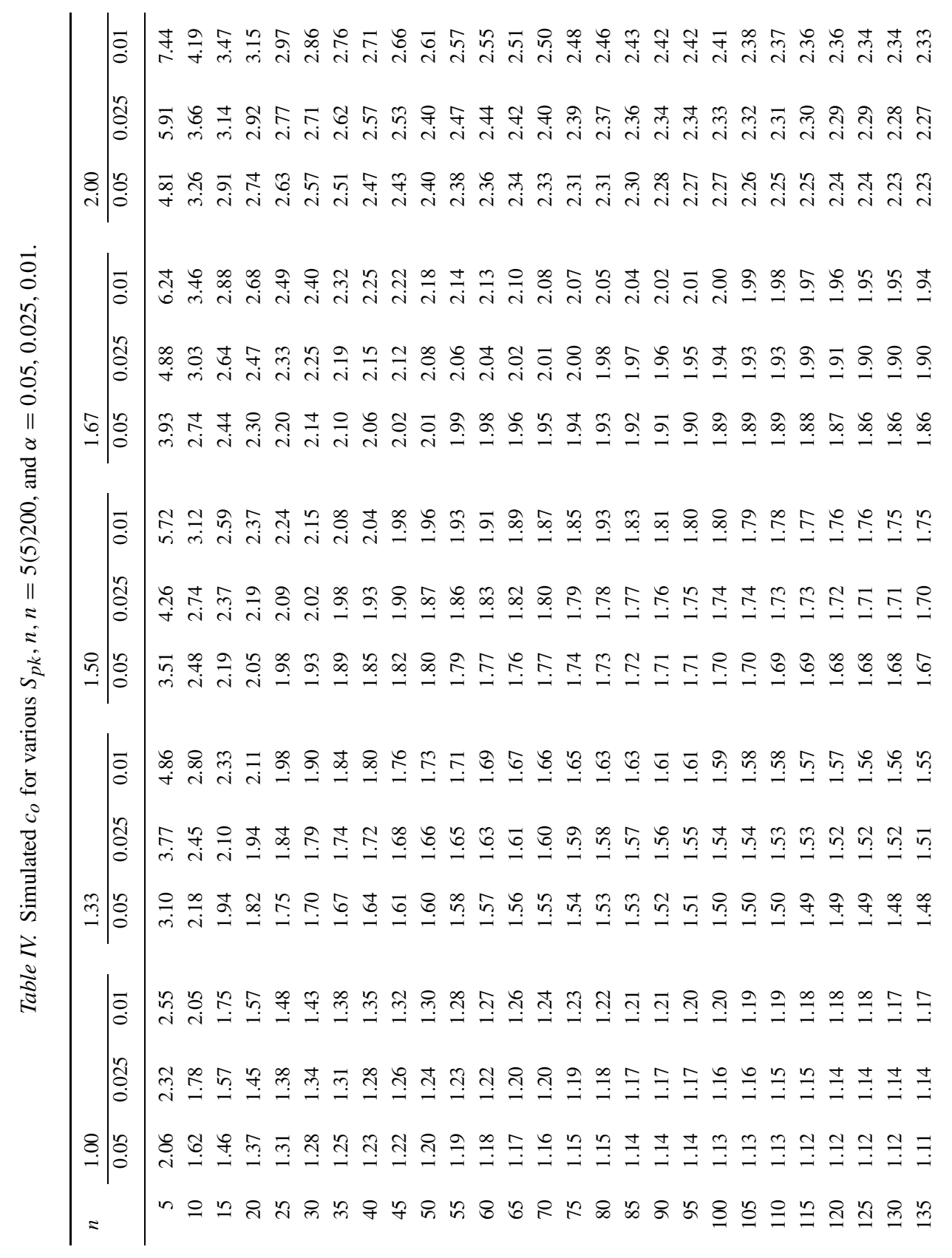




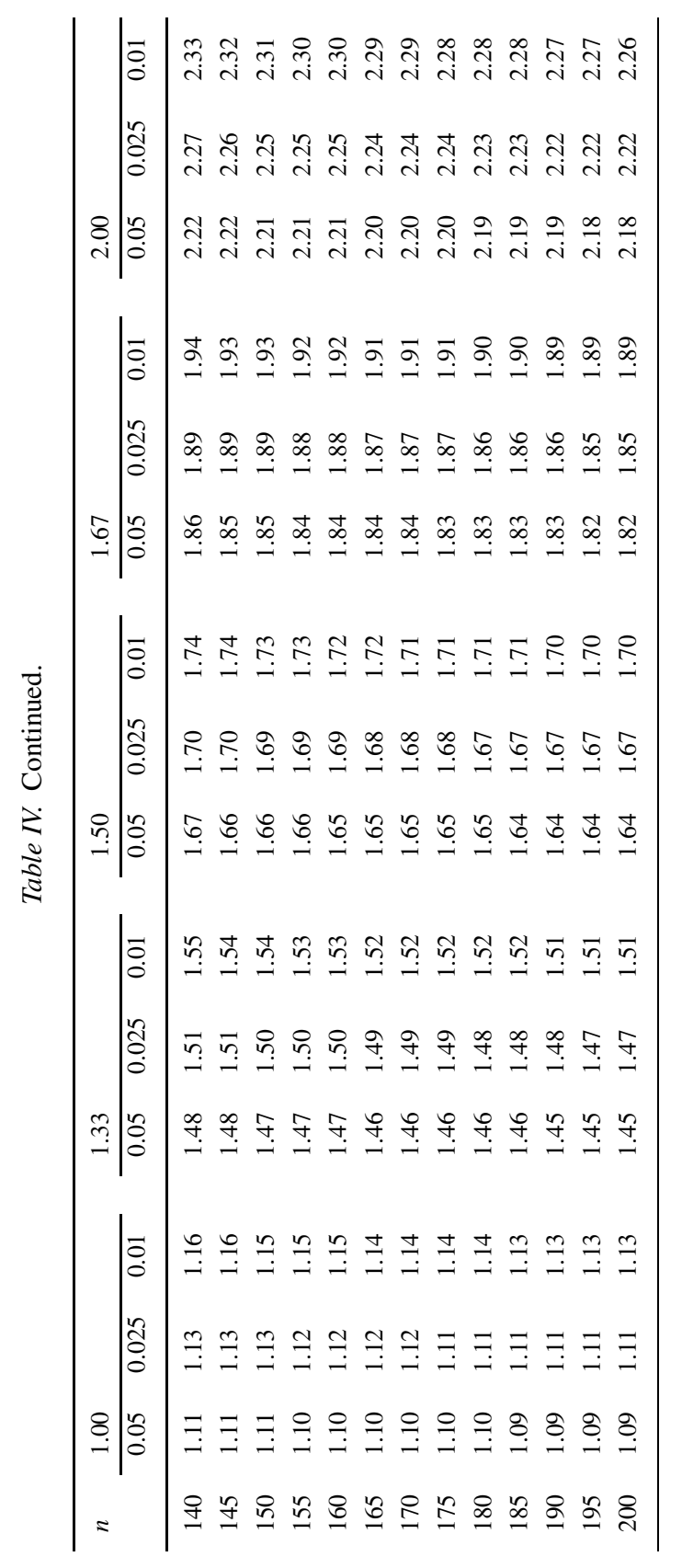


Table $V$. A comparison of $c_{o}$ between the normal approximation and the simulation with $\alpha=0.05$.

\begin{tabular}{rrrrrrrrrrr}
\hline$n$ & 1.00 & 1.33 & 1.50 & 1.67 & 2.00 & & & & & \\
\hline 5 & 1.52 & 2.06 & 2.02 & 3.10 & 2.28 & 3.51 & 2.54 & 3.93 & 3.04 & 4.81 \\
10 & 1.37 & 1.62 & 1.82 & 2.18 & 2.05 & 2.48 & 2.29 & 2.74 & 2.74 & 3.26 \\
15 & 1.30 & 1.46 & 1.73 & 1.94 & 1.95 & 2.19 & 2.17 & 2.44 & 2.60 & 2.91 \\
20 & 1.26 & 1.37 & 1.68 & 1.82 & 1.89 & 2.05 & 2.11 & 2.30 & 2.52 & 2.74 \\
25 & 1.23 & 1.31 & 1.64 & 1.75 & 1.85 & 1.98 & 2.06 & 2.20 & 2.47 & 2.63 \\
30 & 1.21 & 1.28 & 1.61 & 1.70 & 1.82 & 1.93 & 2.03 & 2.14 & 2.43 & 2.57 \\
35 & 1.20 & 1.25 & 1.59 & 1.67 & 1.80 & 1.89 & 2.00 & 2.10 & 2.39 & 2.51 \\
40 & 1.18 & 1.23 & 1.58 & 1.64 & 1.78 & 1.85 & 1.98 & 2.06 & 2.37 & 2.47 \\
45 & 1.17 & 1.22 & 1.56 & 1.61 & 1.76 & 1.82 & 1.96 & 2.03 & 2.35 & 2.43 \\
50 & 1.16 & 1.20 & 1.55 & 1.60 & 1.75 & 1.80 & 1.95 & 2.01 & 2.33 & 2.40 \\
60 & 1.15 & 1.18 & 1.53 & 1.57 & 1.73 & 1.77 & 1.92 & 1.98 & 2.30 & 2.36 \\
65 & 1.14 & 1.17 & 1.52 & 1.56 & 1.72 & 1.76 & 1.91 & 1.96 & 2.29 & 2.34 \\
75 & 1.13 & 1.15 & 1.51 & 1.54 & 1.70 & 1.74 & 1.89 & 1.94 & 2.27 & 2.31 \\
90 & 1.12 & 1.14 & 1.49 & 1.52 & 1.68 & 1.71 & 1.88 & 1.91 & 2.25 & 2.28 \\
110 & 1.11 & 1.13 & 1.48 & 1.50 & 1.67 & 1.69 & 1.86 & 1.89 & 2.22 & 2.25 \\
130 & 1.10 & 1.12 & 1.47 & 1.48 & 1.65 & 1.68 & 1.84 & 1.86 & 2.20 & 2.23 \\
150 & 1.10 & 1.11 & 1.46 & 1.47 & 1.64 & 1.66 & 1.83 & 1.85 & 2.19 & 2.21 \\
\hline
\end{tabular}

Table VI. Sample sizes required for the normal approximation to converge.

\begin{tabular}{rlrrrrrrrrrrr}
\hline & $\alpha$ & 0.10 & 0.09 & 0.08 & 0.07 & 0.06 & 0.05 & 0.04 & 0.03 & 0.02 & 0.01 & \multicolumn{1}{c}{0.00} \\
\hline 1.00 & 0.05 & 124 & 151 & 188 & 241 & 321 & 449 & 670 & 1108 & 2174 & 6053 & 54220 \\
& 0.025 & 176 & 214 & 267 & 342 & 456 & 637 & 951 & 1573 & 3086 & 8594 & 76984 \\
& 0.01 & 248 & 301 & 375 & 482 & 642 & 897 & 1340 & 2216 & 4347 & 12108 & 108455 \\
1.33 & 0.05 & 220 & 266 & 332 & 426 & 568 & 793 & 1185 & 1959 & 3845 & 10707 & 95909 \\
& 0.025 & 312 & 377 & 471 & 605 & 806 & 1126 & 1682 & 2782 & 5458 & 15202 & 136176 \\
& 0.01 & 439 & 531 & 664 & 853 & 1135 & 1586 & 2369 & 3919 & 7690 & 21417 & 191846 \\
1.50 & 0.05 & 279 & 338 & 422 & 542 & 722 & 1009 & 1507 & 2492 & 4890 & 13619 & 121994 \\
& 0.025 & 396 & 480 & 599 & 770 & 1025 & 1432 & 2139 & 3538 & 6943 & 19336 & 173212 \\
& 0.01 & 558 & 676 & 844 & 1084 & 1444 & 2017 & 3014 & 4985 & 9781 & 27241 & 244024 \\
1.67 & 0.05 & 346 & 419 & 523 & 672 & 895 & 1250 & 1868 & 3089 & 6061 & 16881 & 151213 \\
& 0.025 & 491 & 595 & 743 & 954 & 1270 & 1775 & 2652 & 4386 & 8606 & 23968 & 214699 \\
& 0.01 & 692 & 838 & 1046 & 1344 & 1789 & 2500 & 3736 & 6179 & 12124 & 33766 & 302470 \\
2.00 & 0.05 & 496 & 601 & 750 & 964 & 1283 & 1793 & 2679 & 4430 & 8692 & 24211 & 216878 \\
& 0.025 & 704 & 853 & 1065 & 1368 & 1822 & 2545 & 3803 & 6290 & 12342 & 34376 & 307933 \\
& 0.01 & 992 & 1201 & 1500 & 1927 & 2566 & 3585 & 5357 & 8862 & 17388 & 48429 & 433819 \\
\hline
\end{tabular}




\section{An Application Example}

In the following, we present an example of the LCM (liquid crystal module) manufacturing process. The example we investigated was taken from a manufacturing factory making the Liquid Crystal Display Module (LCD Module). The LCD Module is one of the key components used in many high-tech electronic commercial devices for the display function, such as the cellular phone, the PDA (personal digital assistant), the pocket calculator, digital watch, automobile accessory visual displays, and many others. Three key components make the LCD Module functions properly Those include the liquid crystal display, the back lighting, and the peripheral (interface) system.

The mounting technology for the chip-on-glass makes the exposed particle overturned, with the side of circuits facing downward. Then, the electricity conduction is joined between the IC and the panel of the liquid-crystal display through the mounting material. Currently, the mounting technology of the chip-on-glass is the best manufacturing technology for the LCD Module in terms of the mounting density. It is important to note that different mounting material requires different mounting technology of the chip-on-glass.

ACF (Anisotropic Conductive Film) is one of the several developed mounting materials, which is now the most widely used material for the chip-on-glass. For the main bonding process, the bonding precision is an essential process parameter we focused on in our study. We investigated a particular model of the LCD Module product with the upper and the lower specification limits set to USL $=15 \mu \mathrm{m}$, $\mathrm{LSL}=-15 \mu \mathrm{m}$, and the target value is set to $T=0$. If the characteristic data does not fall within the tolerance (LCL, UCL), the lifetime or reliability of the LCD Module will be discounted. To ensure the production quality, the yield index for a particular model we investigated was set to $S_{p k} \geq 1.50$. If the capability requirement fails to be met, the LCD Module product would be seriously affected on its reliability or lifetime.

To test the process performance, we consider the hypothesis testing $H_{0}: S_{p k} \leq$ 1.33 (process is incapable), versus the alternative $H_{1}: S_{p k}>1.33$ (process is capable) with type I error $\alpha=0.05$. According to the process control plan of the COG the random sample data in consecutive four day are collected, which are displayed in Table VII. The 160 sample observations are obtained through the inspection, using the microscope by visual, which were collected eight pieces per every two hours. These 160 observations were justified taken from a stable process (in control), and the characteristic distribution is shown to be approximate normal. The calculated mean and the standard deviation of the 160 sample observations are summarized in the following. Checking Table III we obtain the critical value 1.45 for risk $\alpha=0.05$.

$$
\begin{aligned}
\bar{X} & =0.1754, \quad S=3.1570, \\
\Phi\left(\frac{\mathrm{USL}-\bar{X}}{S}\right) & =0.9999,
\end{aligned}
$$




$$
\begin{aligned}
\Phi\left(\frac{\bar{X}-\mathrm{LSL}}{S}\right) & =0.9999 \\
\hat{S}_{p k} & =\frac{1}{3} \Phi^{-1}\left\{\frac{1}{2} \Phi\left(\frac{\mathrm{USL}-\bar{X}}{S}\right)+\frac{1}{2} \Phi\left(\frac{\bar{X}-\mathrm{LSL}}{S}\right)\right\}=1.5814 .
\end{aligned}
$$

Since the calculated $\hat{S}_{p k}$ from the sample data, 1.58 , is greater than the critical value 1.45 , then we may conclude, with $95 \%$ confidence the process meets the performance requirement $S_{p k}>1.33$. The probability of wrongly judging an incapable process as a capable one is no greater than $5 \%$.

\section{Conclusions}

Process yield is the most common criterion used in the manufacturing industry for measuring process performance. A measurement index, called $S_{p k}$, has been proposed to calculate the yield for normal processes. The index $S_{p k}$ establishes the relationship between the manufacturing specification and the actual process performance, which provides an exact measure on process yield. Unfortunately, the distributional properties of the estimated $S_{p k}$ are mathematically intractable. In this paper, we considered a normal approximation to the distribution of the estimated $S_{p k}$, and investigated its accuracy computationally. We compared the critical values calculated from the approximate distribution with those obtained using the standard simulation technique, for some commonly used quality requirements. Computational results are provided and analyzed. The results indicated that a sample size of $n>150$ is required for the approximation to be accurate. The investigation is useful to the practitioners for making reliable decisions in testing process performance based on the yield.

\section{Appendix}

with(stats):

$\mathrm{n}:=:$ alpha $:=: \mathrm{cp}:=: \mathrm{ca}:=$ :

$\mathrm{x} 1:=3^{*} \mathrm{cp}^{*}(2-\mathrm{ca})$ :

$\mathrm{x} 2:=3^{*} \mathrm{cp}^{*} \mathrm{ca}:$

$\mathrm{p} 1:=$ statevalf[cdf, normald $[0,1] \mathrm{J}(\mathrm{xl})$ :

$\mathrm{p} 2:=$ statevalf[cdf, normald $[0,1]](\mathrm{x} 2)$ :

$\mathrm{p} 3:=(\mathrm{p} 1+\mathrm{p} 2) / 2$ :

spk $:=(1 / 3)^{*}$ statevalf[icdf; normald $\left.[0,1]\right](\mathrm{p} 3)$ :

f1 := statevalf[pdf, normald $[0,1]](\mathrm{x} 1)$ :

$\mathrm{f} 2:=$ statevalf[pdf; normald $[0,1]](\mathrm{x} 2)$ :

f3 := statevalf[pdf, normald[0, 1]](3*spk):

$\mathrm{a}:=2^{\wedge}(-0.5)^{*}\left(\mathrm{x} 1^{*} \mathrm{f} 1+\mathrm{x} 2^{*} \mathrm{f} 2\right)$ :

$\mathrm{b}:=\mathrm{f} 1-\mathrm{f} 2$ :

$\operatorname{var}:=\left(\left(a^{\wedge} 2+b^{\wedge} 2\right) / n\right)^{*}\left(6^{*} \mathrm{f} 3\right)^{\wedge}(-2)$ : 
$\mathrm{c}:=$ statevalf[icdf, normald[0, 1$]](1-\text { alpha })^{*} \operatorname{var}^{\wedge} 0.5: \mathrm{c} 0:=\operatorname{spk}+\mathrm{c}$

\section{References}

Boyles, R. A. (1994). Process capability with asymmetric tolerances. Communication in Statistics: Simulation and Computation 23(3): 615-643.

Lee, J. C., Hung, H. N., Pearn, W. L. \& Kueng, T. L. (2002). On the distribution of the estimated process yield index $S_{p k}$. Quality \& Reliability Engineering International. To appear.

Pearn, W. L., Lin, G. H. \& Chen, K. S. (1998). Distributional and inferential properties of the process accuracy and process precision indices. Communications in Statistics: Theory \& Methods 27(4): 985-1000. 
